\title{
Fast 3D Tomography of C4 Solder Bump by using Xe Plasma Focused Ion Beam
}

\author{
Tomáš Hrnčíŕ, Lukáš Hladík, Jaroslav Jiruše and Filip Lopour
}

TESCAN a.s., Brno, Czech Republic

The Xe plasma ion source integrated on TESCAN FERA3 plasma FIB-SEM instrument offers an extremely high milling and material removal rate, which is important in many application fields, such as failure analysis in the semiconductor industry (e.g. through-silicon vias or flip chip cross sectioning) or MEMS fabrication and analysis [1]. The high resolution of SEM column expands the intrinsic advantages of the Xe plasma ion source. SEM allows observation of the sample during the milling process by utilizing two separate specially designed backscattered electron detectors and a precise endpoint detection based on SEM or FIB image information. With powerful control software, the creation of complex tasks can be automated.

One of these automated tasks is FIB-SEM 3D tomography [2]. Traditionally it has been performed by using Ga beam for sample slicing [2, 3], with the possibility to analyze volumes approximately $10^{3} \mu^{3}$. To obtain the voxel resolution below $10 \mathrm{~nm}$, low FIB current is necessary, which makes the analysis time of the small volume longer. For such voxel sizes FIB has to be operated at probe current below $100 \mathrm{pA}$ [3], but in that case the analyzed volume is limited because of the increased FIB milling time. Analyzed volume can be increased by increasing FIB probe current to units of $\mathrm{nA}$. This allows to obtain still reasonable precision of the milling (voxel size $\sim 100^{3} \mathrm{~nm}^{3}$ ), while keeping the FIB milling time as short as possible. However when the dimension of analyzed volume approaches to $\sim 100^{3} \mu^{3}$, Ga FIB milling time could be several days long. Moreover, the total analysis time is increased by SEM imaging time. Both processes have to be optimized in order to achieve short analysis time.

We have applied the method of 3D tomography to the typical C4 solder bump with the diameter of $100 \mu \mathrm{m}$. Plasma FIB with its 50 times faster sputtering rate against Ga FIB and small spot size at high currents [1] offers much faster processing for such an application. The probe current was set to $100 \mathrm{nA}$ at beam energy $30 \mathrm{keV}$, total analyzed volume $120 \times 120 \times 80 \mu \mathrm{m}^{3}$, cubic voxel edge $234 \mathrm{~nm}$ and voxel resolution $512 \times 512 \times 340$. 3D tomography of such a big volume would be very time consuming and difficult by using the common Ga FIB, the expected milling time being approximately 50 times longer.

All slices were acquired automatically by $3 \mathrm{D}$ tomography data acquisition routine installed on TESCAN FERA3 XMH instrument. Total analysis time was 4.5 hours, of which milling time was 3 hours (512 slices, each $330 \mathrm{~nm}$ thick) and SEM imaging time 1.5 hours. SEM images were saved at pixel dwell time $32 \mu$ s and pixel resolution $512 \times 512$, total saving time for one image was approximately 10 seconds. Secondary electron (SE) detector and In-Beam backscattered electron (BSE) detector (placed inside the SEM column) were used for SEM imaging simultaneously. These detectors are not influenced by a large amount of material redeposited from the sample during the high current plasma FIB milling [1]. InBeam BSE images were chosen for the visualization, because this detector with YAG detecting crystal provides a distinct material contrast and its low acceptance angle is suppressing the topography contrast. ORS Visual ${ }^{\mathrm{TM}}$ software [4] was used for data postprocessing and visualization.

The resulting 3D volume rendering is shown in Figure 1 (a cross section of the model) and Figure 2 (a view on the lower part of the bump). Intermetallic compound (IMC) and under bump metallurgy 
(UBM) layers are clearly visible due to the high material contrast of In-Beam BSE imaging. This method allows observation of cracks or voids in these layers or in the solder bump itself. 3D tomography with plasma FIB seems to have a great potential especially for failure analysis of IC packaging, where it expands the existing range of FIB-SEM applications [5].

\section{References:}

[1] T Hrnčír, F Lopour, M Zadražil, A Delobbe, O Salord, P Sudraud, ISTFA: Conference Proceedings from the 38th International Symposium for Testing and Failure Analysis (2012), p. 26.

[2] L Holzer, F Induthyi, PH Gasser, B Münch and M Wegmann, J. Microsc. 216 (2004), p. 84.

[3] BJ Inkson, M Mulvihill and G Möbus, Scripta Mater. 45 (2001), p. 753.

[4] http://theobjects.com

[5] This work was supported by the EU 7th Framework Programme (Contract No. 280566 - UnivSEM).

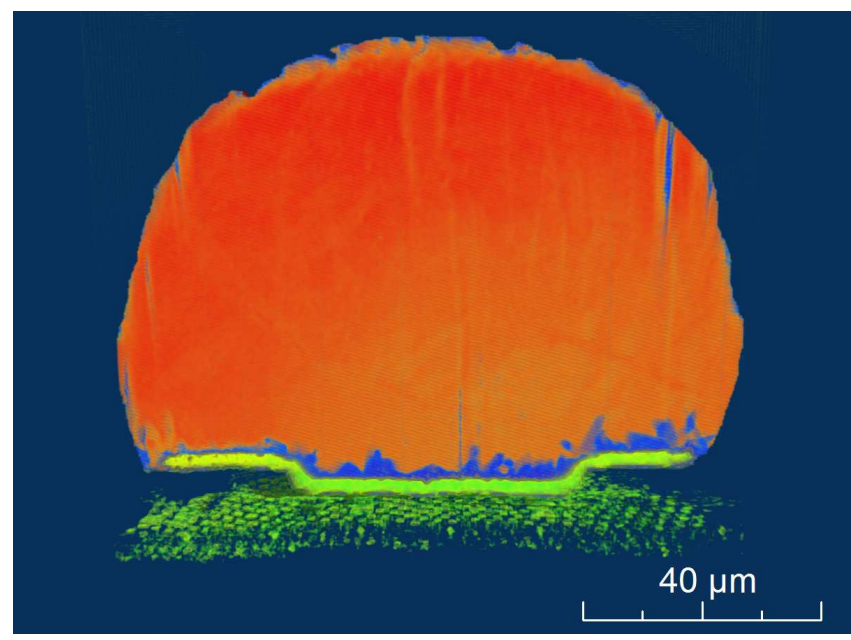

Figure 1. Cross section of 3D model of C4 solder bump. IMC and UBM layers are clearly visible due to the high material contrast of In-Beam BSE imaging.

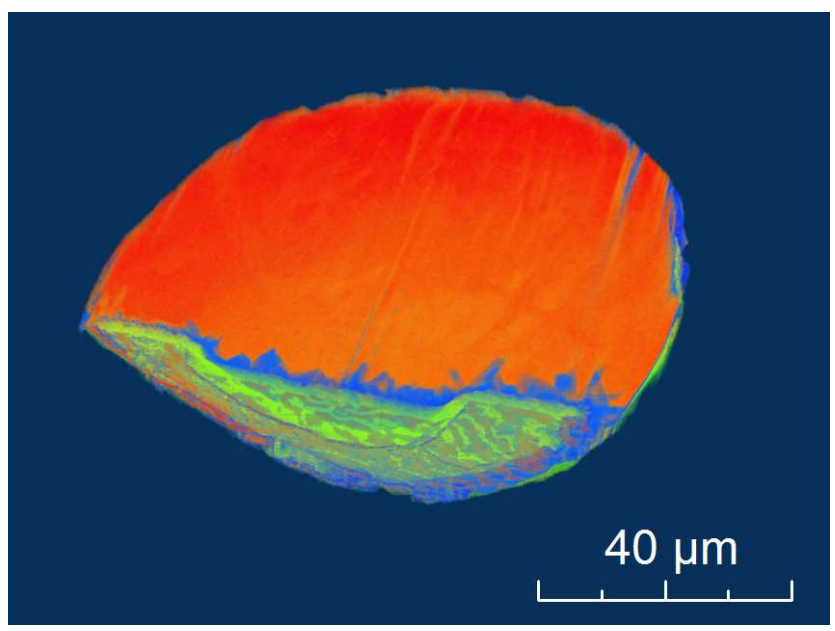

Figure 2. A part of $3 \mathrm{D}$ model of $\mathrm{C} 4$ solder bump, cut approximately in the middle of the bump. Integrated circuit device layers were removed in this image to reveal the shape and the structure of UBM layer. 